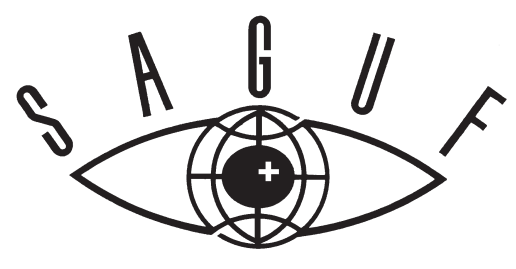

\author{
Schweizerische Akademische \\ Gesellschaft für Umweltforschung \\ und Ökologie \\ Societé Académique Suisse pour \\ la Recherche sur l'Environnement \\ et l'Ecologie \\ Swiss Academic Society for \\ Environmental Research \\ and Ecology
}

\title{
Auf dem Weg zur schweizerischen Biodiversitätsstrategie
}

\author{
Rachel Carson beschrieb bereits 1962 in Der stumme Frühling \\ die Langzeitauswirkungen des chemischen Pflanzenschutzes \\ auf die Artenvielfalt. Artenschutz ist heute dringlicher denn je. \\ Das Forum Biodiversität Schweiz formuliert \\ den Forschungs- und Handlungsbedarf.
}

n der Schweiz leben ähnliche viele Arten wie in manchen europäischen Ländern, die viel größer sind, am Meer liegen und deshalb auch eine vielfältige marine Pflanzen- und Tierwelt aufweisen. Die Aussichten, daß die hohe Artenvielfalt in unserem Land auch in 50 oder 100 Jahren noch existiert, sind allerdings schlecht: Bei den meisten gut untersuchten Organismengruppen gilt mehr als ein Drittel der Arten als selten, bedroht oder als bereits ausgestorben. Nicht nur wildlebende Tiere und Pflanzen, auch viele Nutztierrassen und Kulturpflanzen müssen als gefährdet eingestuft werden (Abbildung 1).
Das Forum Biodiversität Schweiz (siehe Box) veröffentlichte im Oktober 2004 seine umfassende Analyse zur Situation und zu den Trends der Biodiversität in der Schweiz (Baur et al. 2004). Das Werk zeigt zudem auf, wo Defizite im Biodiversitätsschutz liegen, und skizziert Lösungsansätze. Im folgenden legen wir dar, wo wir aus wissenschaftlicher Sicht den dringendsten Forschungs- und Handlungsbedarf sehen.

\section{Das Wissen über Biodiversität fördern} Seit dem Integrierten Projekt Biodiversität im Rahmen des Schwerpunktprogramms Umwelt des Schweizerischen National-

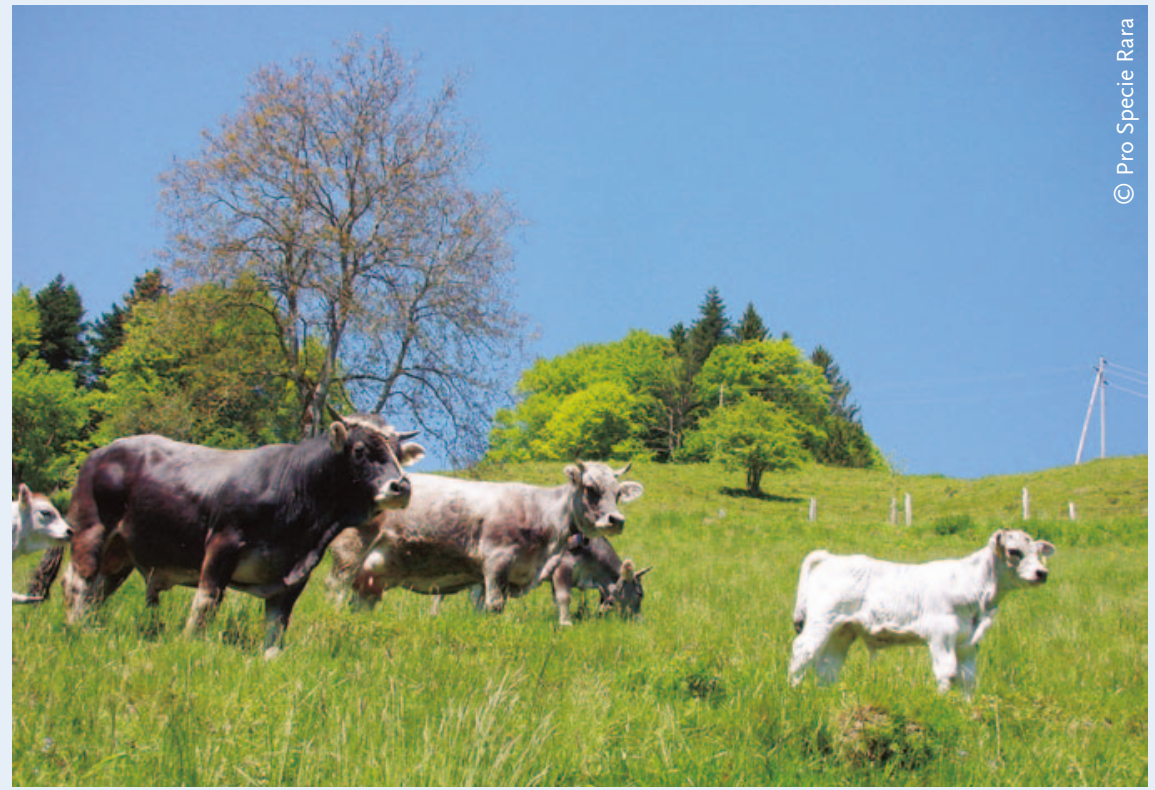

ABBILDUNG 1: Vom Rätischen Grauvieh leben heute dank intensiver Schutzanstrengungen wieder 1500 Tiere in der Schweiz. fonds (1992 bis 2002) wurde in der Schweiz kein großes Biodiversitäts-Forschungsprogramm mehr gefördert. Die wissenschaftliche Bearbeitung folgender Themengebiete ist dringend $\mathrm{zu}$ fördern (siehe auch Forum Biodiversität 2002):

- Erfassung und Messung der Biodiversität: Weiterentwicklung der Systematik verschiedener Organismengruppen (Abbildung 2), Erarbeitung von Biodiversitätsinventaren bisher vernachlässigter Organismengruppen und Habitate, Entwicklung von neuen Methoden zur Erfassung der Biodiversität.

- Einflußfaktoren auf die Biodiversität und geeignete Maßnahmen für die Erhaltung und Förderung der Biodiversität:

relative Wichtigkeit verschiedener natürlicher und anthropogener Einflußfaktoren auf die Biodiversität und mögliche Feedback-Mechanismen (Abbildung 3), Mechanismen evolutiver Prozesse, Korrelationen zwischen verschiedenen $\mathrm{Di}$ versitätsstufen, Abschätzung der Risiken für die Biodiversität, Best-Practice-Methoden für die Erhaltung und nachhaltige Nutzung der Biodiversität.

Kontakt Autor(innen):

Dr. Daniela Pauli | E-Mail: pauli@scnat.ch

Prof. Dr. Bruno Baur|E-Mail: bruno.baur@unibas.ch PD Dr. Irmi Seidl | E-Mail: irmi.seidl@wsl.ch

Kontakt SAGUF: SAGUF-Geschäftsstelle | c/o Claude Théato | ETH Zürich HAD | CH-8092 Zürich | Tel.: +41 / 1 / 6326310 | Fax: +41 / 1 / 6321029 | E-Mail: saguf@env.ethz.ch http://saguf.scnatweb.ch 


\begin{tabular}{l} 
BOX: Das Forum \\
Biodiversität Schweiz \\
Das Forum Biodiversität Schweiz ist \\
eine der thematischen Plattformen der \\
Akademie der Naturwissenschaften \\
(SCNAT). Seine Aufgaben: \\
- Stärkung der Biodiversitätsforschung, \\
- Aufbereitung und Verbreitung \\
wissenschaftlicher Erkenntnisse \\
an ausgewählte Zielgruppen, \\
- Ausbau und Pflege des Dialogs \\
zwischen Wissenschaft und \\
verschiedenen Akteurgruppen, \\
- Einbringen wissenschaftlicher Exper- \\
tise auf nationaler und internationaler \\
Ebene, unter anderem für die \\
Biodiversitätskonvention und \\
- Förderung des öffentlichen Bewußt- \\
seins für den Wert der Biodiversität \\
und die Bedeutung ihrer Erhaltung. \\
Der Beirat des Forum Biodiversität \\
Schweiz umfaßt 23 Wissenschaftler(in- \\
nen) aus vielen Disziplinen sowie Ver- \\
treter(innen) aus Bundesämtern und \\
der Naturschutzpraxis. Der Beirat wird \\
unterstützt durch die Geschäftsstelle in \\
Bern. Daniela Pauli ist Geschäftsleiterin \\
des Forum Biodiversität Schweiz. \\
KONTAKT: \\
Forum Biodiversität Schweiz | \\
Schwarztorstr. 9 | CH-3007 Bern | \\
Tel.: +41 / 31 / 3120275 | \\
E-Mail: biodiversity@scnat.ch | \\
www.biodiversity.ch \\
\hline
\end{tabular}

- Bedeutung der Biodiversität als Grundlage für die menschliche Existenz:

Mechanismen hinter den dokumentierten Zusammenhängen zwischen Biodiversität und Ökosystemfunktionen, monetäre Bewertung von Ökosystemdienstleitungen, Möglichkeiten der Entgeltung der Landeigentümer für die zur Verfügung gestellten Ökosystemdienstleistungen, Eigentumsrechte und $\mathrm{Zu}$ gang zu genetischen Ressourcen.

- Wahrnehmung, Wertschätzung und Bewertung der Biodiversität:

Bedeutung des Konzepts „Biodiversität“ in verschiedenen Kontexten, Quantifizierung und Bewertung von Biodiversität, Entwicklung von Methoden für die Bewußtseinsförderung, Erarbeitung von geeigneten Instrumenten für den Wissenstransfer zwischen Interessengruppen und Regionen.

\section{Wissenstransfer und Dialog ausbauen}

Biodiversitätsrelevante Entscheidungen werden oft ohne ausreichende Kenntnisse über die Auswirkungen auf Pflanzen, Tiere und ihre Lebensräume getroffen. Das nötige Wissen wäre eigentlich vorhanden, doch es steht nicht in geeigneter Form zur Verfügung. Wissenschaftliche Erkenntnisse müssen gesammelt, synthetisiert, verständlich aufbereitet und den zuständigen Stellen in Verwaltung, Naturschutzpraxis und Politik verfügbar gemacht werden. Dies muß im Dialog mit den Adressaten passieren; deren Anliegen und spezifische Fragen beeinflussen sowohl die thematische Fokussierung wie die Art der Aufbereitung der Erkenntnisse entscheidend mit (Fry et al. 2003).

\section{Biodiversität mehrheitsfähig machen}

Umwelt- und insbesondere Naturschutzanliegen haben in den letzten 20 Jahren stark an Bedeutung verloren. Wenn es uns gelingen soll, den Rückgang der Biodiversität bis 2010 zu stoppen - wie dies die europäischen Umweltminister 2003 in Kiew beschlossen haben -, muß die Erhaltung der Biodiversität in allen Politikbereichen und in der Bevölkerung eine hohe Priorität erhalten. Eine politische Interessenvertretung für die Biodiversität, wie sie auch für viele Wirtschaftszweige wie die Landwirtschaft, das Gesundheitswesen oder die Bauwirtschaft besteht, könnte dazu beitragen, daß die Erhaltung und nachhaltige Nutzung der Biodiversität bei politischen Entscheidungen - etwa bei der Subventionspolitik oder der Raumentwicklung eine höhere Priorität erhält.

Biodiversität muß aber auch in der Bevölkerung ein Thema werden. Obwohl sich viele Menschen an einer vielfältigen Natur erfreuen und dabei Erholung finden, ist nur wenigen bewußt, welche Bedeutung Biodiversität hat, in welchem Umfang sie gefährdet ist und welche Möglichkeiten bestehen, sie zu fördern. Damit die Erhaltung der Biodiversität mehrheitsfähig wird, braucht es eine breit angelegte und langfristige Biodiversitätskampagne, welche die unterschiedlichsten Bevölkerungsgruppen gezielt und bedürfnisgerecht anspricht und für die biologische Vielfalt begeistert.

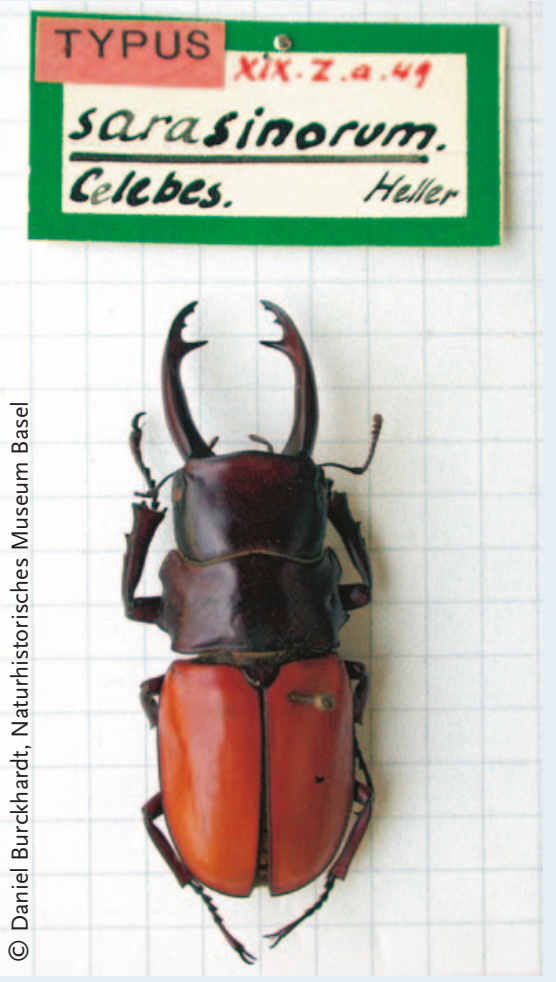

ABBILDUNG 2: In Schweizer Museen gibt es eine große Anzahl an Typusbelegen wie diesen Hirschkäfer (Odontolabis sarasinorum) aus Sulawesi. Typen bilden die materielle Referenz von wissenschaftlichen Artnamen. Museen brauchen zusätzliche finanzielle Ressourcen, um Sammlungen unterhalten und Informationen zur Biodiversität digitalisieren und im Internet zugänglich machen zu können.

\section{Vollzug bestehender Gesetze verbessern}

Die Inhalte der Gesetze für den Natur- und Landschaftsschutz decken die Forderungen für die Erhaltung und nachhaltige Nutzung der Biodiversität ab. Für den Vollzug dieser Regelungen sind in der Schweiz die Kantone zuständig. Der Vollzug ist jedoch vielfach mangelhaft, etwa im Auen- und im Moorschutz. Damit die Kantone die an sie delegierten Naturschutzkompetenzen besser wahrnehmen können, sind den kantonalen Verwaltungen ausreichende finanzielle und personelle Ressourcen zur Verfügung zu stellen. Zudem müßte der Bund Sanktionsmöglichkeiten zur Hand haben, um dort eingreifen zu können, wo die Kantone den Vollzug zu wenig ernst nehmen.

Im Gewässerschutz sind Mikroverunreinigungen besonders problematisch, darunter Schwermetalle, Arzneimittel, Hor- 
mone, Desinfektionsmittel und Waschmittelbestandteile. Für alle relevanten Stoffe sind Qualitätsstandards festzulegen. Besonders schwer abbaubare Verbindungen müssen vor allem bei Massenchemikalien durch biologisch vollständig abbaubare Substanzen ersetzt werden. In landwirtschaftlich genutzten Gebieten muß das Gewässerschutzgesetz konsequent umgesetzt werden.

\section{Land- und Forstwirtschaft gezielter auf die Erhaltung und Förderung der Biodiversität ausrichten}

Der landwirtschaftlichen Nutzung kommt eine Schlüsselrolle bei der Erhaltung der Biodiversität in der Schweiz zu. Mit der in den 1990er Jahren eingeleiteten Reform der Agrarpolitik ist die Schweiz auf dem richtigen Weg zu einer nachhaltigen Landwirtschaft. Noch aber ist mit ökologischen Ausgleichsflächen das Ziel, die Biodiversität im Kulturland zu erhalten und zu fördern, nicht erreicht. Erste Evaluationen sprechen immerhin von einem „moderat positiven Effekt“ (Herzog et al. 2005). Für landwirtschaftliche Nutzungstypen oder auch Kleinstrukturen - etwa Trockenmauern oder stehende Kleingewässer -, die die Biodiversität besonders fördern, sind zusätzliche Anreize zu schaffen: einerseits durch höhere Direktzahlungen, andererseits durch attraktive Absatzmöglichkeiten von Produkten, zum Beispiel durch die Einbeziehung von Biodiversitätskriterien in Produktlabels.
Im Schweizer Wald ist die Biodiversität heute noch weniger gefährdet als in anderen Lebensräumen. Jetzt wird das Waldgesetz revidiert. Dabei ist dringend darauf zu achten, daß der Erhaltung der Biodiversität auch in Zukunft große Bedeutung beigemessen wird. So sind vom Bund national bedeutende Waldflächen festzulegen und Vorranggebiete auszuweisen, in denen die Erhaltung und Förderung der Biodiversität an erster Stelle steht. Zudem muß die Erhaltung der Biodiversität auf der ganzen Fläche ein wichtiges Kriterium für die Waldbewirtschaftung sein.

\section{Bestehende Instrumente verbessern und ausbauen}

Artenreiche Lebensräume sind oft nur noch in Regionen zu finden, in denen Naturschutzgebiete bestehen, während sie aus ähnlichen Gegenden ohne Naturreservate fast vollständig verschwunden sind. Allerdings sind die bestehenden Reservate $\mathrm{zu}$ klein und $\mathrm{zu}$ isoliert, um langfristig das Überleben der darin wohnenden Tiere, Pilze und Pflanzen zu sichern. Sie müssen mit ausreichenden Pufferzonen versehen werden, brauchen Bewirtschaftungs- und Pflegepläne und sollten miteinander und mit anderen naturnahen Landschaftselementen verbunden werden. In Landschaften, die für die Biodiversität besonders wertvoll sind, sind zusätzlich Vorrangflächen einzurichten, in denen die Erhaltung und Förderung der besonderen Lebensräume und ihrer Arten höchste Priorität hat.
Bei stark gefährdeten Arten reichen generelle Maßnahmen zur Förderung der Biodiversität wie der ökologische Ausgleich in der Landwirtschaft oder die Einrichtung von Schutzgebieten oft nicht aus, um die Bestände langfristig zu erhalten oder sogar wieder aufzubauen. Sie benötigen Artenförderungsprogramme mit art- und problemgerecht zugeschnittenen Maßnahmen.

\section{Biodiversität überwachen}

Ein Biodiversitätsmonitoring muß problematische Bestandsentwicklungen frühzeitig aufdecken können, Warnungen herausgeben und Maßnahmen auslösen. Das schweizerische Biodiversitätsmonitoring (BDM-CH) gilt dafür international als Vorzeigebeispiel. Das BDM-CH sollte um weitere Komponenten der Biodiversität erweitert werden, unter anderem zur Gewässerbiodiversität. Für einige Organismengruppen bestehen zusätzlich detailliertere Datensammlungen, die das BDM-CH für ausgewählte Fragestellungen ergänzen beispielsweise für die Überprüfung des Ziels, bis 2010 den Rückgang der Biodiversität zu stoppen. Die Schweiz mit ihrer umfangreichen und wissenschaftlich fundierten Datengrundlage sollte die Chance nutzen, hier eine Vorreiterrolle zu übernehmen.

Die Wirkung von Naturschutzmaßnahmen kann nur mit einer gezielten Erfolgskontrolle nachgewiesen werden. Sie dient außerdem dazu, allenfalls nötige Korrekturen der Maßnahmen einzuleiten. Zur Zeit
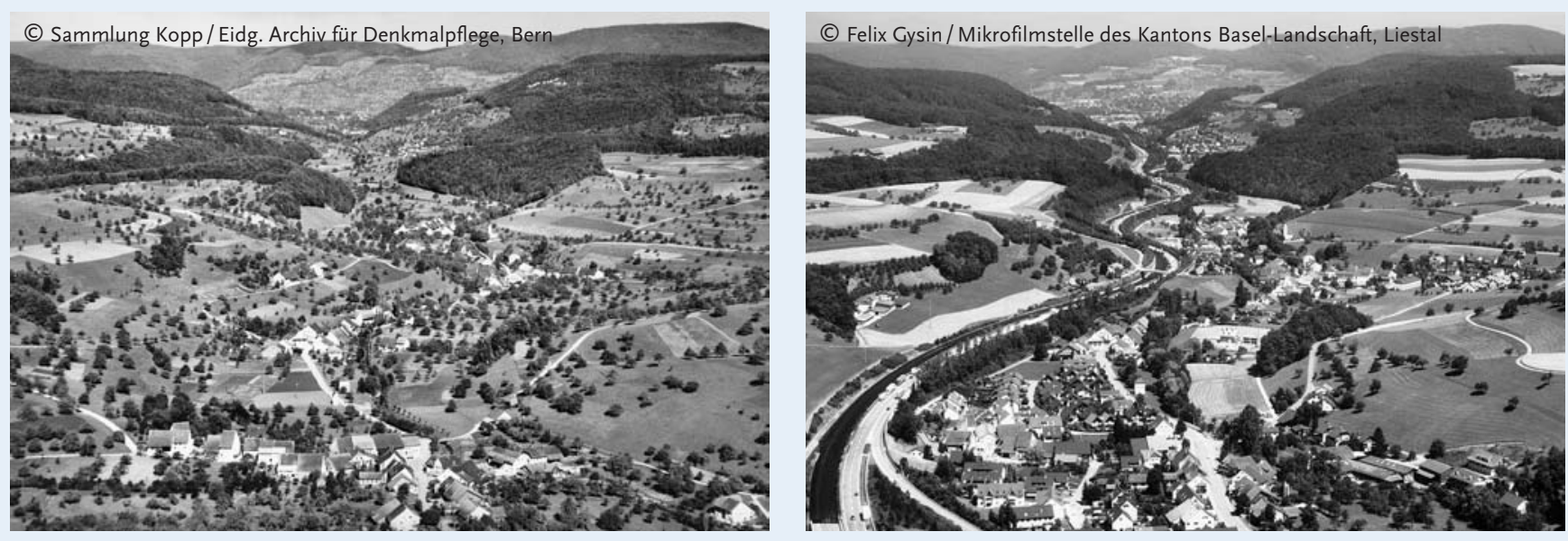

ABBILDUNG 3: Ein Vergleich des Diegtertals (Kanton Basel-Landschaft) zwischen 1955 (links) und 1995 (rechts) zeigt die Veränderung der Landschaft und damit wichtige Ursachen für den lokalen Biodiversitätsverlust in diesen 40 Jahren. Die Nationalstraße zerschneidet das Tal in zwei Teile, die meisten einzeln stehenden Bäume sind verschwunden, die Kleinparzellierung der landwirtschaftlichen Flächen wurde aufgegeben und der Siedlungsraum stark ausgedehnt. 
politische ökologie

Die Zeitschrift für Querdenkerinnen und Vordenker

\section{Werte schöpfen}

Ideen für nachhaltiges

Konsumieren und Produzieren

Globalisierungskritiker, Verbraucherpolitik und Umweltlobby wollen den Weltmarkt stärker regulieren. Auf der anderen Seite zeigt sich König Kunde von Kampagnen für nachhaltigen Konsum unbeeindruckt. Die politische ökologie ${ }^{94}$ zeigt neue Strategien für Politik, NGOs und

Unternehmen.

_Brauchen Verbraucher einen Benimmkurs? _Wie lässt sich der Markt als Instrument der Nachhaltigkeit nutzen?

_Was halten Jugendliche von Bio und Fairtrade?

Mit Beiträgen von A. Troge, E. Müller, L. Reisch, G. Scherhorn, B. Pötter, K. Ankele, T. v. Hardenberg, C. Erdmenger u.v.m.

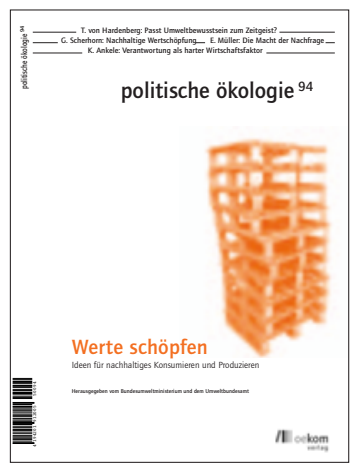

Stillen Sie Ihre Neugier!

_Fordern Sie Heft 94 an _12,00 EUR (inkl. Versand)/19,20 SFR _ISBN 3-936581-91-6

_oekom verlag

_Fax +49/(0)89/54 418449

_www.oekom.de

neugier@oekom.de besteht die Tendenz, die Erfolgskontrollen im Naturschutz abzubauen. Das Gegenteil muß jedoch der Fall sein: Die bisherigen Erfolgskontrollen - zum Beispiel bei den Inventaren wertvoller Biotope - sind unbedingt weiterzuführen und auf andere Instrumente des Biodiversitätsschutzes auszudehnen.

\section{Eine nationale Biodiversitätsstrategie entwickeln}

Mit der Unterzeichnung der Biodiversitätskonvention haben sich Staaten verpflichtet, die Entwicklung der Biodiversität zu planen. In der Schweiz existieren dafür zwar wichtige Ansätze, wie etwa das Landschaftskonzept Schweiz. Bisher fehlt aber eine Biodiversitätsstrategie, die alle Politikbereiche einbezieht und klare, überprüfbare Ziele für die Biodiversität setzt. Die Autor(inn)en der Strategie werden Vorstellungen entwickeln müssen, welche Teile der Biodiversität in der Schweiz zu erhalten sind, sie müssen zudem

- Zieleinheiten mit Indikatoren

(Populationen, Unterarten, Arten, Ökosystemfunktionen, Lebensräume, Landschaften) definieren und in ihrer Priorität festlegen,

- Zielgrößen vorgeben für die zu erhaltenden Organismen und Flächen und diese regional aufteilen,

- fachlich abgestützte Maßnahmen für die Erhaltung und nachhaltige Nutzung der Biodiversität nennen und

- Wege aufzeigen, wie diese Maßnahmen umgesetzt werden können.

Jedes Land trägt in erster Linie eine besondere Verantwortung für den Teil der Biodiversität, der aus biogeographischen oder kulturhistorischen Gründen besonders ausgeprägt oder in seinem Vorkommen einmalig ist. Dazu gehören alle endemischen Arten sowie europa- oder weltweit bedrohte Arten, von denen ein Land einen großen Anteil der Population beherbergt. Doch die Biodiversitätskonvention verpflichtet jedes Land, der globalen Biodiversität durch Tätigkeiten unter seiner hoheitlichen Kontrolle außerhalb seiner Grenzen Sorge zu tragen. Dies betrifft unter anderem den Import von Produkten, die Vergabe von Exportrisikogarantien und die
Entwicklungszusammenarbeit. Die nationale Biodiversitätsstrategie muß deshalb auch die Rolle der Schweiz für die globale Biodiversität bestimmen.

Bereits 1998 forderten renommierte Wissenschaftler eine Biodiversitätsstrategie für die Schweiz (Suter et al. 1998). Inzwischen sind wir einen großen Schritt weitergekommen. Nach der Publikation der wissenschaftlichen Grundlagen für die Strategie im Oktober 2004 (Baur et al. 2004) wurde im November 2004 die Parlamentarische Gruppe „Biodiversität und Artenschutz" gegründet, der sich bis heute 64 Eidgenössische Parlamentarier(innen) aus allen Fraktionen angeschlossen haben. Damit hat die Biodiversität im Parlament eine Interessenvertretung erhalten. Am 9. Dezember 2004 reichten 74 Parlamentarier(innen) ein Postulat ein, das vom Bundesrat fordert, eine Biodiversitätsstrategie für die Schweiz zu erarbeiten.

Wir danken Herrn K. M. Tanner, dem Autoren des Buches Tanner, K. M. 2001. AugenBlicke. Bilder zum Landschaftswechsel im Baselbiet. Liestal: Verlag des Kantons Basel-Landschaft, für die freundliche Abdruckgenehmigung der Fotos in Abbildung 3.

Der Vorstand der SAGUF hat das Forum Biodiversität Schweiz der Akademie der Naturwissenschaften (SCNAT) zu diesem Beitrag eingeladen.

\section{Literatur}

Baur, B., P. Duelli, P. J. Edwards, M. Jenny, G. Klaus, I. Künzle, S. Martínez, D. Pauli, K. Peter, B. Schmid, I. Seidl, W. Suter. 2004. Biodiversität in der Schweiz: Zustand, Erhaltung, Perspektiven. Wissenschaftliche Grundlagen für eine nationale Strategie. Herausgegeben von Forum Biodiversität Schweiz. Bern: Haupt Verlag.

Forum Biodiversität Schweiz. 2002. Visions in Biodiversity research - Towards a new integrative biodiversity science. www.biodiversity.ch/ publications (abgerufen 04.07.2005).

Fry, P., I. Seidl, C. Théato, A. Kläy, F. Bachmann. 2003. Vom Wissenstransfer zum Wissensaustausch: Neue Impulse für den Boden- und Biodiversitätsschutz in der Landwirtschaft. GAIA 12: 148-150.

Herzog, F., S. Birrer, J. Derron, P. Duelli, S. Pearson, L. Pfiffner, T. Walter. 2005. Artenvielfalt im Kulturland: Welchen Beitrag leisten ökologische Ausgleichsflächen? HOTSPOT 11: 8-9.

Suter, W., M. Bürgi, K. C. Ewald, B. Baur, P. Duelli, P. J. Edwards, J.-B. Lachavanne, B. Nievergelt, B. Schmid, O. Wildi. 1998. Die Biodiversitätsstrategie als Naturschutzkonzept auf nationaler Ebene. GAIA 7: 174-183. 